\title{
ROCs obtained with two signal intensities presented in random order, and a comparison between yes-no and rating ROCs ${ }^{1}$
}

DAVID S. EMMERICH ${ }^{2}$

HEARING AND COMMUNICATION LABORATORY, INDIANA UNIVERSITY

Two experiments are presented. The first experiment investigated the effect of mixing signals of two different intensities within the same blocks of trials up on receiver operating characteristics (ROCs) obtained with the rating procedure. The ROCs obtained when the signals of differing intensity were mixed in blocks of trials were found to be quite similar to those obtained when only one signal intensity was presented in any given block of trials. In the second experiment ROCs were determined by having subjects adopt different decision criteria on different blocks of trials in a yes-no experiment. The data points obtained in this experiment fell quite close to the ROCs obtained from the same subjects with the rating procedure in the first experiment.

One of the important contributions of the theory of signal detectability to psychophysics has been the introduction of the receiver operating characteristic (ROC) as a methodological tool. The ROC is the function which relates the probability of a correct detection (or hit) to the probability of an incorrect response indicating that a signal was present (or false alarm), in a signal-detection experiment. Use of the ROC permits the experimenter to separate the sensitivity of the observer from nonsensory factors such as response bias.

There are several ways to obtain an ROC. Consider an experiment in which a single observation interval is defined on each trial, and on some trials a signal is added to the noise background during the observation interval. Now provide the subject with two buttons to push: a "yes" button to indicate a decision that a signal was presented, and a "no" button to indicate a decision that no signal was presented. The subject is instructed to push one button or the other after each trial. For obvious reasons, this type of experiment is often called a singleinterval yes-no experiment. If the subject is induced to adopt different criteria on different blocks of trials for how certain he must be before he presses the "yes" button, then we can estimate the probabilities of hits and false alarms from these blocks of trials, and plot points on an ROC.

Another way of obtaining an ROC requires the subject to maintain a number of such criteria simultaneously. On each trial of a single-interval experiment the subject makes a decision as to how certain he is that a signal was or was not presented, and then expresses this decision by making a rating response. For example, Egan, Schulman, and Greenberg (1959) determined points on ROCs by having subjects push one of four buttons on each trial to express their degree of certainty that a signal was presented. The authors of this study found that the ROCs determined by the rating method were very similar to those determined by having the same subjects adopt different criteria on different blocks of trials. The rating procedure is more efficient, however, permitting an ROC to be determined with a much smaller number of trials.

Watson, Rilling, and Bourbon (1964) extended the rating procedure by employing a response manipulandum which permitted the definition of a large number of categories. Rather than requiring the subject to push one of a number of buttons on each trial, Watson, Rilling, and Bourbon had the subject position a slider which had a continuous range. In recording and analyzing the data obtalned in this experiment the experimenters divided up the range of the slider into 36 categories.

The first experiment presented here compares ROCs obtained with different signal intensities mixed in the same blocks of trials, to ROCs obtained when the same signal intensities are presented in separate blocks of trials. If the subject in the single-interval rating task is able to use the rating scale reliably, and successive judgments are independent, then the ROCs passed through the obtained points when signal intensities are mixed in certain blocks of trials should be quite similar to those obtained when the same intensities are presented in separate blocks of trials.

Tucker, Evans, and Jeffress (1967) have recently presented ROCs obtained with seven signal intensities mixed in the same blocks of trials. They did not obtain ROCs with the same signal intensities presented in separate blocks of trials, however.

Blocker (1965) investigated the effect of multiple signal intensities on performance in a two-interval forced-choice experiment. Her data indicate that in this situation the effect of mixing different signal intensities is small, when a comparison is made with performance on the same task with the signal intensities presented separately.

A second experiment presented here compares ROCs obtained by having subjects adopt different criteria on different blocks of trials in a single- 
interval yes-no experiment to ROCs obtained with a single-interval rating procedure. As mentioned earlier, Egan, Schulman, and Greenberg (1959) present evidence that the two procedures lead to similar results. Swets, Tanner, and Birdsall (1961) report an experiment in which, for a visual task, ROCs obtained by the rating method were found to be slightly closer to the chance line than those obtained with the single-interval yes-no procedure. When the experiment was replicated, however, the authors could find no consistent differences between the ROCs obtained by the two methods. In these experiments the a priori probability of signal presentation was 0.50 on all trials. Green and Swets (1966) point out that when a mechanical slider is used additional variability may be introduced into the ROC data. The second experiment presented here addresses itself to this possibility by comparing ROCs determined by having subject employ three different criteria in the yes-no paradigm, to those obtained with a mechanical rating-scale device which permitted responses to be partitioned into 20 categories. As in the above experiments, the a priori probability of signal presentation was 0.50 on all trials. A recent study by Markowitz and Swets (1967) indicates that there are systematic differences between yesno and rating ROCs. In their study, however, subjects were induced to change criteria in the yes-no part of the experiment by varying the a priori probability of signal presentation, rather than by varying instructions to the subjects.

\section{GENERAL PROCEDURE}

In this section those aspects of the experimental situation which were common to both experiments are described. Two experimental paradigms used in the experiments are also described. Later references to these paradigms should be understood to imply the specific details (e.g., time intervals, a priori probabilities) mentioned below. Additional procedural details relating to each particular experiment will be introduced in subsequent sections.

Both experiments involved the detection of a 400 cps sinusoid presented in a background of wideband white Gaussian noise by means of a headset employing Permoflux PDR-10 earphones. The noise was obtained from a Grason-Stadler 455-B noise generator, and was continuously present at a spectral level of approximately $45 \mathrm{~dB}$ re 0.0002 microbar. The sinusoidal signal was gated without regard to phase, and transients were determined by the response characteristics of the earphones. The signal and the noise stimuli were presented diotically. Four Ss were run at the same time. Each was seated in an individual sound-deadened booth.

Before each block of trials, a signal preview, or tone demonstration, was provided for the Ss. These tone demonstrations consisted of several brief pre- sentations of the sinusoidal signal at an intensity $10 \mathrm{~dB}$ higher than that at which it would be presented during the block of trials, followed by several brief presentations at the same intensity at which it would be presented during the block of trials.

\section{The single-inferval yes-no experiment}

In this paradigm only one observation interval is defined on each trial. The S's task is to indicate whether or not a signal was presented by pressing one of two buttons (a "yes" button and a "no" button) during the response interval. By varying instructions it is possible to induce the $S$ to adopt different criteria for how certain he must be that a signal was presented before he will press the "yes"' button.

A trial in the single-interval yes-no experiment consisted of the following events: a $500 \mathrm{msec}$ warning light, a $500 \mathrm{msec}$ pause, a $350 \mathrm{msec}$ observation interval (indicated by a light), a $2000 \mathrm{msec}$ response interval, and a final $500 \mathrm{msec}$ pause. The probability that a signal would be presented during the observation interval was 0.50 .

If a signal was presented during the observation interval it was initiated $100 \mathrm{msec}$ after the beginning of the interval and terminated at the end of the interval, resulting in a signal duration of 250 msec. Feedback lights indicating whether or not a signal was present could be illuminated during the final pause. The S's responses were recorded on electromechanical counters.

\section{The single-interval rating experiment}

In this paradigm the events defining a trial are of exactly the same form as those defining a trial in the single-interval yes-no experiment. In the singleinterval rating experiment to be discussed later the duration of the response interval was $2500 \mathrm{msec}$ rather than 2000 msec; with this exception the various intervals of time were the same in both single-interval paradigms. The S's task in the rating experiment is quite different from the task in the single-interval yes-no experiment, however. In the rating experiment the $S$ is not asked to make a simple binary decision on each trial, but instead is asked to rate each trial as to its "signal-likeness."

A special response manipulandum was constructed in the attempt to make the $S^{\prime} S$ task as simple as possible, while permitting his responses to be collected and analyzed by automated equipment. This manipulandum consisted of a pushbutton mounted on a small carrier which rode on a pair of milled tracks. This pushbutton projected through a slot in a metal plate which permitted it to be moved horizontally through a range of approximately $13.5 \mathrm{in.}$ In back of the metal plate a series of sections of polished brass tubing was mounted (out of the S's view) in such a way that depression of the S's push- 
button resulted in a mechanical contact with one and only one of the brass sections. In this manner the range of the sliding pushbutton, though apparently continuous to the $S$, was actually divided into 20 discrete categories of equal length. The mechanical contact of the S's pushbutton with a brass section resulted in the conduction of an electrical pulse through an encoding network of diodes to a set of storage relays, from which the encoded information representing the S's response was ultimately punched onto paper tape.

The center of the slot in the metal plate was marked and the $S$ was instructed to return the sliding pushbutton to the center of the slot at the end of each trial. The $\mathrm{S}$ was instructed to use a press of the pushbutton at the extreme right end of the slot to indicate that he was very certain that a signal had been presented, a press of the pushbutton at the extreme left end of the slot to indicate that he was very certain that a signal had not been presented, and positions closer to the center to indicate intermediate degrees of certainty. The $S$ was asked to use the entire range of the slider to communicate his judgments of certainty that a signal (or noise alone) had been presented, if possible. The $S$ was also told that an equivalent way to describe his task was for him to consider it one of rating each trial as to its "signal-likeness" or "noise alone -likeness."

In summary, we emphasize that the single-interval rating experiment is formally identical to the single-interval yes-no experiment, except that a more complex response is required of the $S$. Because of the increased complexity of the required response and the fact that it takes an appreciable amount of

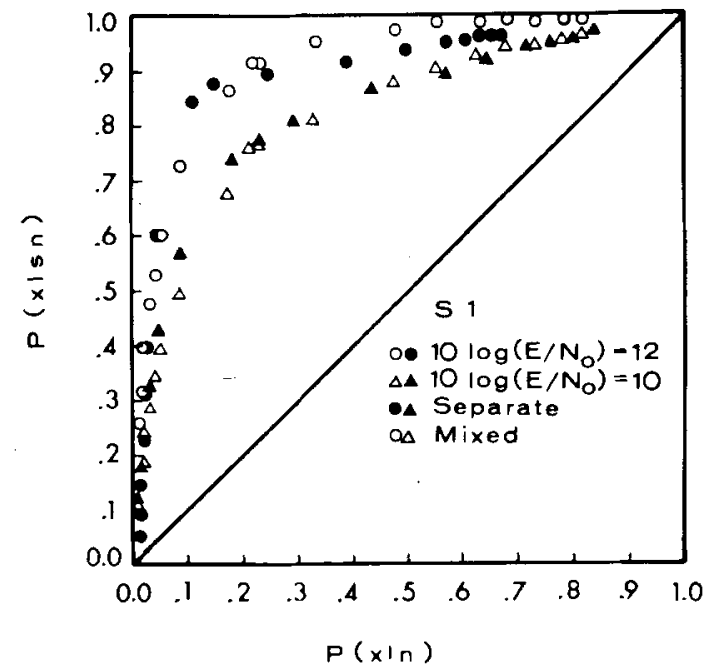

Fig. 1. RoCs obtained from Subject 1 . Solid symbols represent data from blocks of trials in which only one signal intensity was presented. Open symbols represent data from blocks of trials in which both signal intensities were presented.

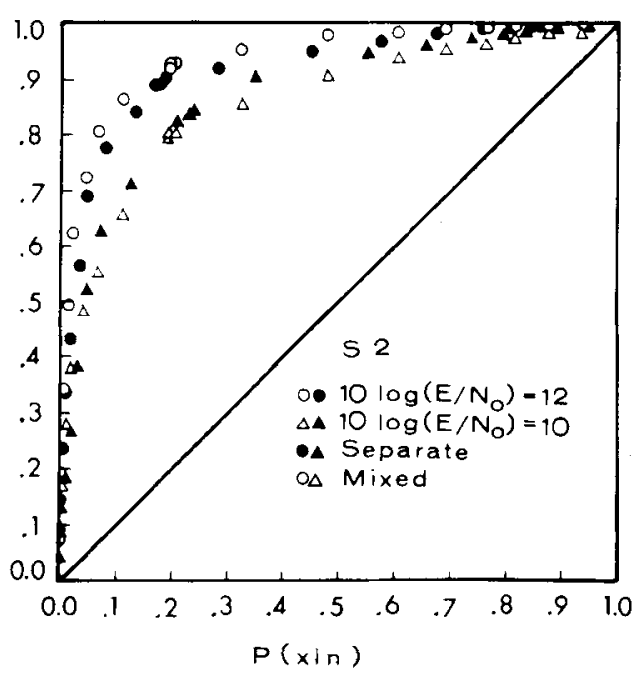

Fig. 2. ROCs obtained from Subject 2. See caption for Fig. 1.

time merely to move the slider six or seven inches (half the length of the slot in which it travels), the duration of the response interval in the rating experiment was increased by $500 \mathrm{msec}$.

\section{EXPERIMENT 1}

If the $S$ is able to use the rating-scale device reliably to convey his judgments in the single-interval rating experiment, and if successive judgments are independent, we would expect that presenting signals with differing values of $10 \log \left(\mathrm{E} / \mathrm{N}_{0}\right)$ on different trials of the same block would have little effect on the resulting ROC curves. That is, the following two procedures should yield ROCs which are quite similar in form: (1) present signals differing in $10 \log \left(\mathrm{E} / \mathrm{N}_{\mathrm{o}}\right)$ in the same blocks of trials, and calculate separate ROCs for each value of $10 \log \left(E / N_{0}\right)$; (2) present signals differing in $10 \log \left(\mathrm{E} / \mathrm{N}_{\mathrm{o}}\right)$ in separate blocks of trials, and calculate ROCs for each value of $10 \log \left(\mathrm{E} / \mathrm{N}_{\mathrm{o}}\right)$. Experiment 1 was conducted to test this prediction.

\section{Procedure}

A group of four young women with clinically normal hearing was employed for this experiment. None of these Ss had previously participated in an auditory experiment.

All the data in this experiment were obtained with the presentation of trial-by-trial feedback. Prior to the experiment three days of practice were given. During the first day of practice, a two-interval forced-choice paradigm was used in order to familiarize the Ss with the detection task, and to give the $\mathrm{E}$ an indication of how well they could detect signals at several different values of $10 \log \left(E / N_{o}\right)$. During the next two days of practice the singleinterval rating paradigm was used. 


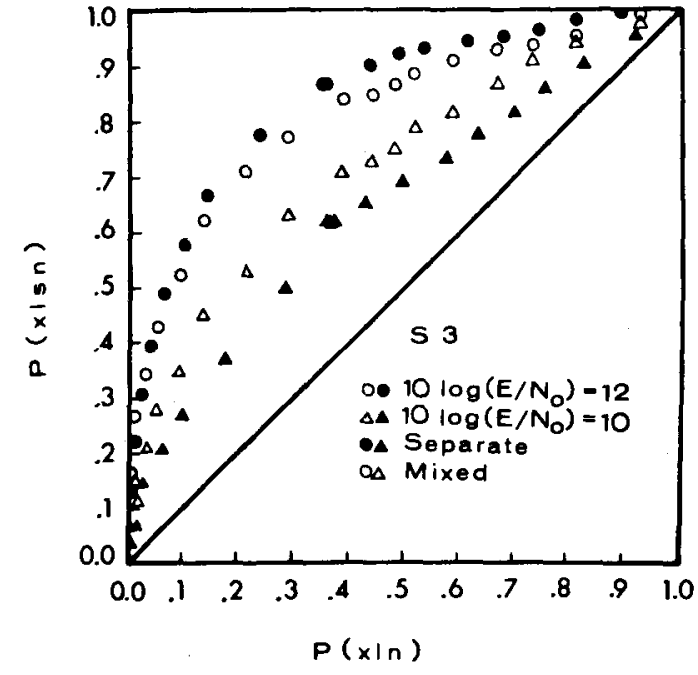

Fig. 3. ROCs obtained from Subject 3. See caption for Fig. 1 .

The experiment lasted for seven and one-half sessions. A session was divided into two segments which were separated by a 6-min rest period. Each segment consisted of a 40-trial practice block followed by three 80-trial data blocks. These four blocks were separated by 2 -min rest periods. Before each block a tone demonstration was provided for the Ss. On each trial of the experiment the probability of the occurrence of a signal was 0.50 . On some days, however, if a signal occurred, the probability that the value of $10 \log \left(E / N_{O}\right)$ associated with it was 12 was the same as the probabllity that the value of $10 \log \left(\mathrm{E} / \mathrm{N}_{\mathrm{O}}\right)$ associated with it was 10 (1.e., 0.50). On the other days, the $10 \log$ $\left(E / N_{o}\right)$ of the signal remained fixed at a particular value. The single-interval rating paradigm was used throughout the experiment.

During the first session of the experiment the $10 \log \left(\mathrm{E} / \mathrm{N}_{\mathrm{O}}\right)$ of the $400 \mathrm{cps}$ tone was 12. During the second session the $10 \log \left(\mathrm{E} / \mathrm{N}_{\mathrm{O}}\right)$ of the tone was 10. For the following three and one-half sessions these two values of $10 \log \left(E / \mathrm{N}_{0}\right)$ were mixed randomly (each with probability 0.25 of occurrence) within each block. During the next session the value of $10 \log \left(\mathrm{E} / \mathrm{N}_{\mathrm{o}}\right)$ was again 12 , and in the final session $10 \log \left(E / N_{0}\right)$ was 10.

\section{Results and Discussion}

The data obtained in this experiment were a serles of ratings which could range from 1 to 20. A rating of 1 on a particular trial indicated that the $S$ was very certain that a signal had been presented, and a rating of 20 indicated that the $S$ was very certain that noise alone had been presented. Ratings falling between 1 and 20 indicated intermediate degrees of certainty. These data were analyzed, using a method described by Egan and Clarke (1962), to obtain the

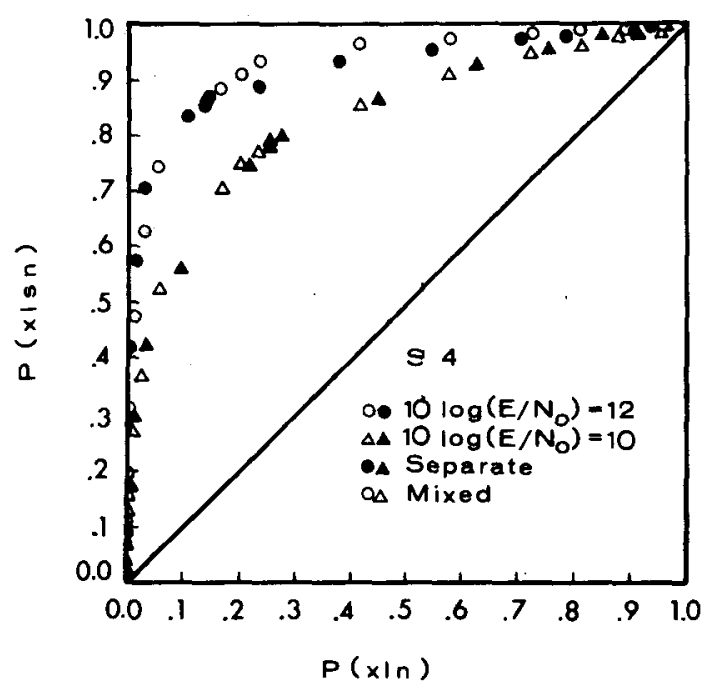

Fig. 4. RoCs obtained from Subject 4. See caption for Fig. 1.

ROCs presented in Figs. 1 through 4. Each ROC represents the cumulative proportion of trials resulting in a rating $r$ or less given signal, plotted against the cumulative proportion of trials resulting in a rating $r$ or less given nolse alone, as $r$ varies from 1 to 20 . The final point on the ROC thus must be the point $(1,1)$. This point is not plotted on the ROCs of Figs. 1 through 4.

When the two different signal intensities were presented in the same blocks of trials, the responses to the different signal intensities were analyzed separately to obtain estimates of the hit rates for different response criteria. (The noise-alone trials from these blocks were used to obtain common estimates of the false-alarm rates for both signal intensities. Thus the ROCs obtained from blocks containing both signal intensities share the same $x$-coordinates.) As can be seen from Figs. 1 through 4, the ROCs determined with the two values of $10 \mathrm{log}$ $\left(\mathrm{E} / \mathrm{N}_{\mathrm{O}}\right)$ mixed randomly in the same blocks of trials are quite similar in form to the ROCs determined

Table 1.

Areas under ROCs determined in Experiment 1 with two values of $10 \log \left(E / N_{0}\right)$ mixed in the same hlocks of trials, and with these values of $10 \log \left(E / N_{0}\right)$ presented in separate blocks of trials.

\begin{tabular}{llll} 
& & \multicolumn{2}{c}{$10 \log \left(E / N_{0}\right)$} \\
& & 10 & 12 \\
\hline Subject 1 & Mixed & .815 & .912 \\
Subject 2 & Separate & .824 & .894 \\
& Mixed & .860 & .943 \\
Subject 3 & Separate & .876 & .920 \\
Subject 4 & Mixed & .715 & .813 \\
& Separate & .642 & .842 \\
Average & Mixed & .833 & .927 \\
& Separate & .827 & .922 \\
& Mixed & .806 & .899 \\
& Separate & .792 & .894 \\
\hline
\end{tabular}


with the two values of $10 \log \left(E / N_{0}\right)$ presented in separate blocks of trials.

To facilitate comparison of the ROCs presented in Figs. 1 through 4, the areas under these ROCs are presented in Table 1 . In order to compute these areas, adjacent points on each ROC were connected by stralght lines. The areas under the ROCs obtained with the two different experimental conditions (two signal intensities mixed in blocks, and two signal intensitles presented in separate blocks) are generally quite similar in magnitude for both values of $10 \log \left(\mathrm{E} / \mathrm{N}_{\mathrm{o}}\right)$ employed.

The fact that the ROCs obtained in this experiment were essentially the same for a given signal intensity (whether it was presented by itself or in blocks of trials also containing another signal intensity) indicates that the Ss were able to use the rating-scale device reliably and consistently to convey trial-by-trial information about the stimulus. In other words, the data of this experiment support the view that the stimulus has a high degree of control over the S's behavior in the single-interval rating task.

\section{EXPERIMENT 2}

As mentioned earlier, it is possible to generate points on an ROC by using the single-interval yes-no procedure and asking the $S$ to vary his criterion for how certain he must be that a signal was present before he presses the yes button. Using this technique, experimenters generally have the $S$ adopt three, or at most four, different criteria during the course of an experiment. It is of interest to know if the points obtained from a given $S$ with this technique lie close to the points obtained from the same $S$ with the use of the rating device described earlier. Experiment 2 investigated this possibility. In this experiment, the single-interval yes-no procedure was employed to determine three points on an ROC

Table 2. Points on the ROCs obtained in Experiment 2 by having subjects vary their decision criteria

\begin{tabular}{lcc} 
& Hit Rate & False Alarm Rate \\
\hline Subject 1 & Strict Criterion & \\
Subject 2 & .518 & .046 \\
Subject 3 & .610 & .064 \\
Subject 4 & .458 & .134 \\
\hline & .558 & .068 \\
Subject 1 & Medium Criterion & \\
Subject 2 & .763 & .270 \\
Subject 3 & .791 & .206 \\
Subject 4 & .727 & .481 \\
\hline Subject 1 & .772 & .220 \\
Subject 2 & Lax Criterion & .472 \\
Subject 3 & .886 & .454 \\
Subject 4 & .952 & .534 \\
\hline
\end{tabular}

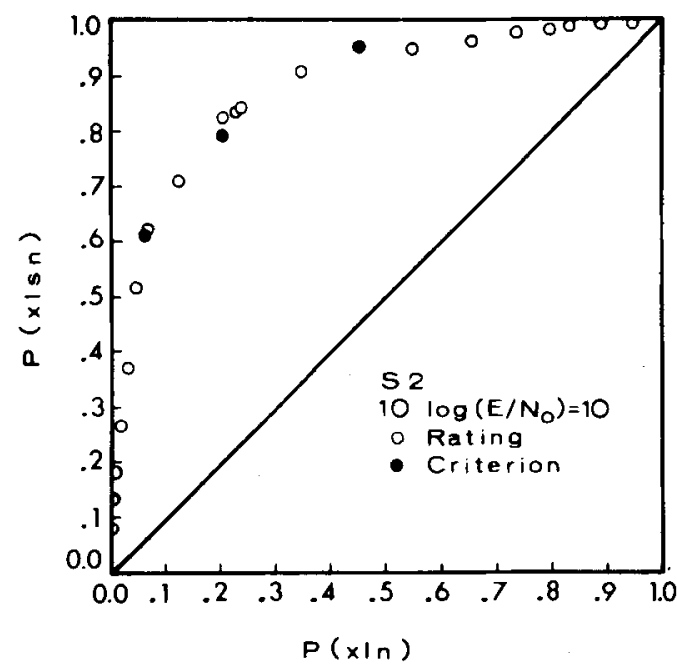

Fig. 5. ROCs obtained from Subject 2. Filled circles represent data obtained by having the subject adopt different decision criteria. Open circles represent data obtained with the rating technique.

for each of four Ss. The results of this experiment may be compared to the appropriate data of Experiment 1 .

\section{Procedure}

The same four Ss were employed in this experiment as were employed in Experiment 1. Experiment 2 lasted for two sessions and each session had a duration of approximately $1.5 \mathrm{~h}$. Each day's session was divided into three segments separated by $6-\mathrm{min}$ rest periods. Each segment consisted of a 20-trial practice block followed by three 80-trial data blocks. These four blocks of trials were separated by 2-min rest perlods. Before each block a tone demonstration was provided for the Ss. All data of Experiment 2 were obtained with $10 \log \left(E / N_{O}\right)$ equal to 10 , and with the presentation of trial-by-trial feedback. The single-interval yes-no procedure was employed.

One session of practice was given before the experiment began, in order to familiarize the Ss with the single-interval yes-no procedure and to establish meanings for the criteria "strict," "medium," and "lax" which the Ss were subsequently asked to adopt. The $\mathbf{E}$ decided that a "strict" criterion should result in a proportion of presses of the yes button when noise alone was presented which fell between 0.05 and 0.10 , and that a "lax" criterion should result in a proportion of presses of the yes button when a signal was presented which fell between 0.90 and 0.95. It was decided that a "medium" criterion should result in the sum of these two proportions lying within 0.07 of 1.00 . If the proportions calculated at the end of a block of trials met the appropriate specifications above, Ss were told that their per- 
formance was "good." If a S's performance did not meet these specifications, the $S$ was asked to be either "more strict" or "more lax."

During the first session of the experiment proper the following procedure was followed: during the first third of the session (one practice block and three 80-trial data blocks) the Ss were told to adopt a "strict" criterion, during the next third the Ss were told to adopt a "medium" criterion, and during the last third the Ss were told to adopt a "lax" criterion. In the second session this procedure was reversed: The Ss were told to adopt a "lax" criterion during the first third of the session, a "medium" criterion during the next third, and a "strict" criterion during the final third.

\section{Results and Discussion}

The coordinates of the three points determined for each $S$ are presented in Table 2. The proportions presented in this table are each derived from six blocks of 80 trials. Figure 5 presents the threepoint ROC obtained from one of the $S$ s in this experiment, and an ROC determined for the same $S$ and the same stimulus conditions in Experiment 1. (The ROC from Experiment 1 was determined with only one signal level being presented in each block of trials.) As can be seen from the figure, there is good agreement between the ROC obtained with a single-interval yes-no procedure and the ROC obtained with a single-interval rating procedure. The data from this $S$ are considered representative: no systematic differences were observed for any of the Ss. This result is in agreement with the findings of Egan, Schulman, and Greenberg (1959), and supports the view that both types of ROC provide the same kind of information. As previously mentioned, Markowitz and Swets (1967) have found systematic differences between yes-no and rating ROCs. In this study the Ss were induced to change criteria in the yes-no paradigm by varying the a priori probability of signal presentation, rather than by varying instructions to the Ss. The a priori probability of signal presentation was always 0.50 in the rating paradigm, however. The authors suggest that this procedural difference may have led to the systematic differences which they found between yes-no and rating $\mathrm{ROCs}$.

\section{References}

Blocker, A. S. The use of multiple signal levels in a study of the relationship between signal level and the detectability index, $d^{\prime}$. Unpublished masters thesis, University of Texas, 1965

Egan, J. P., \& Clarke, F. R. Psychophysics and signal detection. Tech. Rep. No. ESD-TDR-62-305, Contract No. AF 19(628)-266, 1962. This report is reproduced as Chapter 5 in $\mathbf{J}$. B. Sidowski (Ed.), Experimental methods and instrumentation in psychology. New York: McGraw-Hill, 1966.

Egan, J. P., Schulman, A. I., \& Greenberg, G. Z. Operating characteristics determined by binary decisions and by ratings. $J$. Acoust. Soc. Amer., 1959, 31, 768-773

Emmerich, D. S. Receiver operating characteristics determined under several interaural conditions of listening. Dissertation and Technical Report, Hearing and Communication Laboratory, Indiana University, Bloomington, Indiana, 1967

Green, D. M., \& Swets, J. A. Signal detection theory and psychophysics. New York: Wiley, 1966.

Markowitz, J., \& Swets, J. A. Factors affecting the slope of empirical ROC curves: comparison of binary and rating responses. Percept. \& Psychophys., 1967, 2, 91-100.

Swets, J. A., Tanner, W. P., Jr., \& Birdsall, T. G. Decision processes in perception. Psychol. Rev., 1961, 68, 301-340.

Tucker, A., Evans, R. B., \& Jeffress, L. A. ROC curves for multiple-signal levels in a detection task. Paper read at Acoust. Soc. Amer., New York, April, 1967.

Watson, C. S., Rilling, M. E., \& Bourbon, W. T. Receiver-operating characteristics determined by a mechanical analogue to the rating scale. J. Acoust. Soc. Amer., 1964, 36, 283-288.

\section{Notes}

1. Based upon Emmerich (1967), a doctoral dissertation and Technical Report of the Hearing and Communication Laboratory, Indiana University, Bloomington, Indiana. The data of the experiments presented here, as well as those of two additional experiments, are included in tables and appendices of this technical report. 2. Present address: Department of Psychology, State University of New York at Stony Brook, Stony Brook, New York 11790.

3. I wish to thank Dr. James P. Egan and Dr. Donald E. Robinson for their guidance both in the preparation of this paper and in the conduct of the research presented in it. The research presented in this paper was conducted while the author was a University Fellow of Indiana University. The research was sponsored in part by the Air Force Office of Scientific Research, Office of Aerospace Research, under Grant AF-AFOSR-548-67, and in part by a Dissertation Research Grant from Indiana University. The laboratory in which the research was conducted was constructed with funds granted by the National Science Foundation.

(Accepted for publication August 25, 1967.) 